\title{
La narcolepsie : un modèle d'étiologie multifactorielle
}

La narcolepsie associe une somnolence diurne excessive et des accès de cataplexie. Cette maladie est presque toujours associée à la présence de l'antigène HLA-DR2 dont la fréquence n'est que de $22 \%$ dans la population témoin. Son développement suivrait l'exposition d'un individu génétiquement prédisposé à des facteurs extrinsèques physiques ou psychologiques. Les relations entre la présence de l'antigène HLA-DR2 du complexe majeur d'histocompatibilité et le désordre neurochimique responsable des signes cliniques restent inconnues.

\section{Jacques Montplaisir Gaétan Poirier Roger Godbout Raymonde Marinier}

Subventionné par le conseil de recherches médicales du Canada et le fond de la recherche en santé du Québec.

\section{ADRESSE}

J. Montplaisir, G. Poirier, R. Godbout, R. Marinier: centre d'étude du sommeil, hôpital du Sacré-Cœur, 5400 bd Gouin Ouest, Montréal (Québec), H4J IC5, Canada. $m / s n^{\circ} 4$ vol. 4 , avril 88 a classification établie par l'association of sleep disorders centers reconnaît quatre catégories diagnostiques de troubles du sommeil dont l'une est l'hypersomnie. Cette dernière regroupe la narcolepsie, l'hypersomnie périodique ou syndrome de Kleine-Levin, et les hypersomnies secondaires à des troubles psychiatriques ou respiratoires ainsi qu'au syndrome des mouvements périodiques des membres au cours du sommeil. D'autres formes d'hypersomnies sont liées à l'usage de l'alcool ou de divers agents pharmacologiques.

La narcolepsie est une forme spécifique d'hypersomnie reconnue comme entité pathologique distincte depuis plus de 100 ans [1]. L'intérêt d'étudier cette maladie dépasse largement le champ spécifique de la physiopathologie des troubles du sommeil. En effet, la narcolepsie peut nous fournir un modèle de maladie à facteurs étiologiques multiples, nous guidant dans l'étude de plusieurs autres maladies sans lien apparent avec cette condition. C'est ainsi que nous tenterons de démontrer comment des facteurs biochimiques, génétiques et environnementaux peuvent interagir pour engendrer la narcolepsie.

\section{Aspects cliniques}

Symptomatologie. La narcolepsie se caractérise par la présence de deux symptômes, une somnolence diurne excessive (SDE) et des accès d'atonie musculaire ou attaques de cataplexie. La présence simultanée de ces deux 


\section{RÉFÉRENCES}

1. Passouant P. Historical note : doctor Gelineau (1828-1906). Narcolepsy centennial. Sleep $1981 ; 4$ : 241-6.

2. Godbout R, Montplaisir J. All-day performance on normal and narcoleptic subjects. Sleep $1986 ; 9$ : 200-4.

3. Montplaisir J, Godbout R. Nocturnal sleep of narcoleptic patients : revisited. Sleep 1986 ; 9 : 159-61.

4. Wittig R, Zorick F, Piccione P, Sicklesteel J, Roth T. Narcolepsy and disturbed nocturnal sleep. Clin Electroencephalogr 1983 ; $14: 130-4$.

5. Montplaisir J, Walsh J, Lapierre G. Nocturnal sleep of hypersomniacs: a positive correlation between REM fragmentation and cataplexy. In : Chase MH, Kripke DF; Walter PL, eds. Sleep Research, vol. 10. Los Angeles: Brain Information Service, 1981: 218

6. Broughton R. Evoked potentials and the assessment of EDS in narcolepsy. Abstract from the 5th International Congress of Sleep Research. Copenhague : Gen, 1987 : 426.

7. Aguirre M, Broughton R J. Complexevent-related potentials (P300 and CNV) and MSLT in the assessment of excessive daytime sleepiness in narcolepsy-cataplexy. Electroencephalogr Clin Neurophysiol 1987 ; 67 : 298316.

8. Mefford IN, Baker TL, Boehme R, et al.. Narcolepsy: biogenic amine deficit in an animal model. Science 1983 ; 220 : 629-32.

9. Kaitin KI, Kilduff TS, Dement WC. Evidence of excessive sleepiness in canine narcoleptics. Electroencephalogr Clin Neurophysiol $1986 ; 64: 447-54$

10. Montplaisir J, De Champlain J, Young SN, et al. Narcolepsy and idiopathic hypersomnia: biogenic amines and related compounds in CSF. Neurology 1982; 32 compounds symptômes est essentielle au diagnostic de la maladie.

La SDE est pratiquement constante et entraîne une dégradation des performances psychomotrices [2]. Elle atteint parfois son paroxysme sous la forme de poussées incoercibles de sommeil. Le caractère typique de ces «siestes » est leur grand pouvoir réparateur, même lorsque celles-ci ne durent que quelques minutes. La SDE peut également expliquer la présence de comportements automatiques où l'individu poursuit, en dépit d'un niveau diminué de vigilance, des activités motrices qui lui sont familières. En plus du fait de comporter un risque important d'accident, la SDE constitue un handicap social évident.

La cataplexie correspond à une paralysie brève et plus ou moins complète des muscles somatiques, sans perte de la conscience, déclenchée par des émotions comme le rire, la colère ou la surprise. Les patients narcoleptiques apprennent souvent à contrôler ces émotions ou à éviter le plus possible les situations susceptibles de les engendrer.

Une majorité de malades présente également des paralysies du sommeil, c'est-à-dire une incapacité complète de se mouvoir, survenant à l'endormissement ou au réveil. Ce symptôme particulièrement angoissant s'accompagne souvent d'hallucinations qui peuvent être de nature auditive, visuelle ou somesthésique*. Finalement, le sommeil nocturne des patients narcoleptiques est perturbé : il est allégé, entrecoupé d'éveils répétés et les cauchemars sont fréquents [3, 4].

Examens complémentaires. Les études de laboratoire ont montré que les sommeils diurne et nocturne des patients narcoleptiques sont perturbés de façon caractéristique, et plus particulièrement le sommeil paradoxal. Le patient narcoleptique s'endort beaucoup plus rapidement qu'un sujet sain et le stade de sommeil paradoxal apparaît habituellement en moins de dix minutes (contre 90 minutes chez des sujets normaux). La pré- sence de ces endormissements directs en sommeil paradoxal est essentielle au diagnostic de la maladie. Le sommeil paradoxal du narcoleptique est par ailleurs fragmenté, c'est-à-dire parsemé d'éveils ou de passages à d'autres stades du sommeil. Cette fragmentation est peut-être associée au développement de la cataplexie [5]. La durée moyenne des différents stades du sommeil est toutefois comparable à celle des sujets normaux.

L'électroencéphalogramme des patients narcoleptiques est normal et l'étude des potentiels évoqués a mis en évidence des modifications inconstantes [6]. Des études récentes montrent toutefois des différences significatives entre certaines mesures de potentiels liés à l'événement (P 300) chez des narcoleptiques et des sujets normaux [7]. Cependant, il est probable que ces changements sont le reflet de l'hypersomnolence de ces malades et non des marqueurs biologiques spécifiques de la maladie.

Évolution de la maladie. Une étude rétrospective menée chez 61 de nos patients a montré que l'évolution de la narcolepsie varie considérablement en fonction des individus. Chez tous les malades, la somnolence et les poussées de sommeil furent les premiers symptômes à apparaître, à un âge variant de 5 à 54 ans (moyenne: 23,1 ans) suivis de la cataplexie, en moyenne huit ans plus tard. La figure 1 (page 241) montre que plus l'apparition de la SDE est tardive, plus la cataplexie se développe rapidement. Les paralysies du sommeil, les hallucinations hypnagogiques $^{* *}$ et les comportements automatiques sont apparus à divers stades évolutifs et étaient

\footnotetext{
* Somesthésique : se dit d'une sensation d'origine corporelle (chaud, froid, douleur, tact pression, sensibilité proprioceptive). Les hallucinations somesthésiques sont plus rares dans la narcolepsie et sont généralement proprioceptives. Le patient a alors l'impression, par exemple, que son corps s'élève au-dessus du lit ou s'enfonce dans le matelas.

** Hypnagogiques: qui surviennent à l'endormissement.
} 


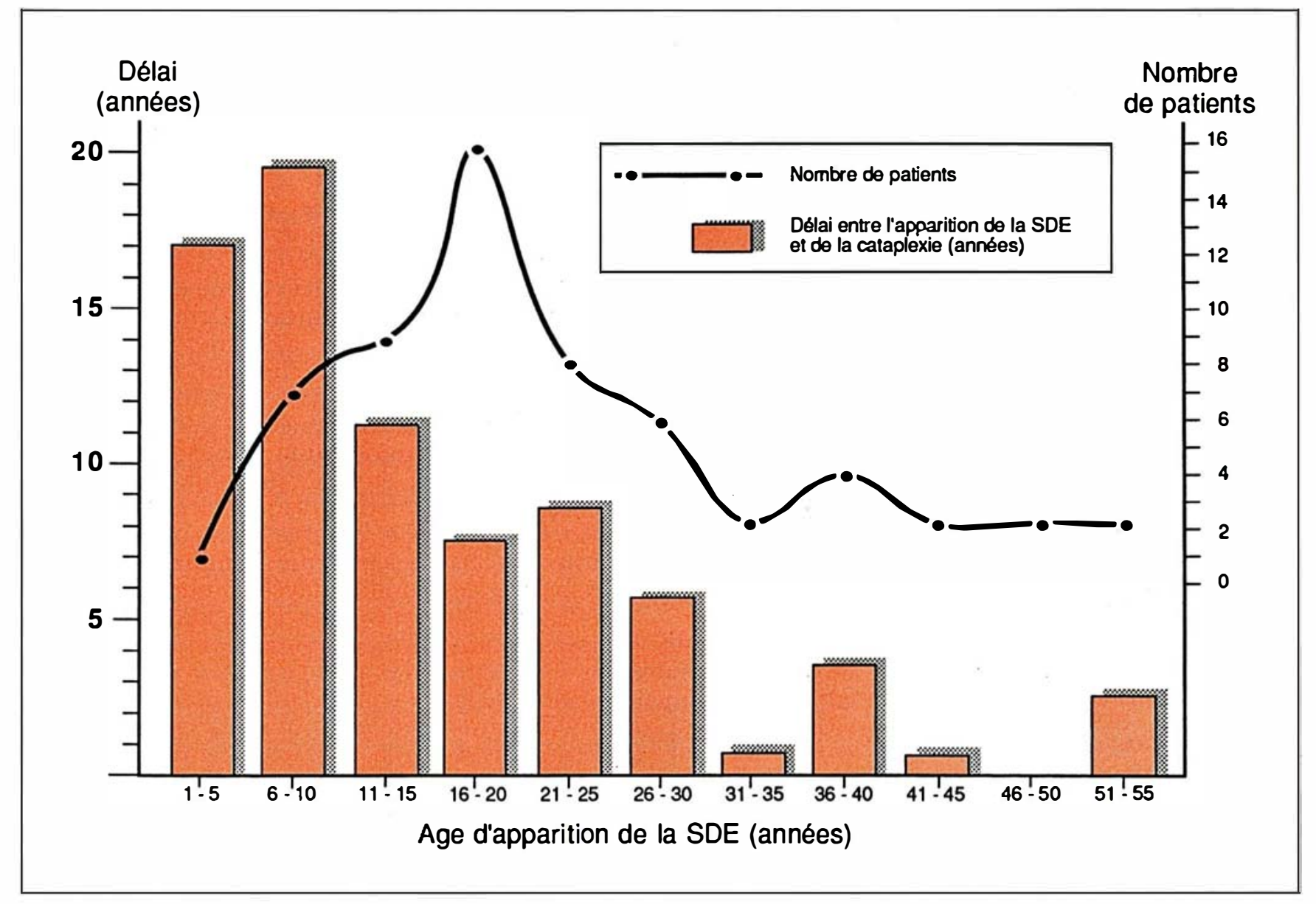

Figure 1. Apparition des symptômes de narcolepsie chez 58 malades.

totalement absents dans $35 \%$ des cas.

\section{Données biologiques et expérimentales}

L'étiologie de la narcolepsie est encore mal connue. De nombreuses recherches ont porté sur les aspects neurochimique et génétique de la maladie et sur les événements entourant l'apparition des premiers symptômes.

De l'animal à l'homme. Des modèles naturels canins de la narcolepsie ont été étudiés principalement pour la cataplexie. En résumé, la cataplexie canine pourrait dépendre d'une hypersensibilité cholinergique (augmentation du nombre de récepteurs muscariniques dans le tronc cérébral et effet thérapeutique des $\mathrm{m} / \mathrm{s} n^{\circ} 4$ vol. 4 , avril 88 anticholinergiques muscariniques) et d'une dépression des systèmes mono-aminergiques (diminution des taux de renouvellement des amines)[8]. Bien que la cataplexie soit réelle chez les chiens narcoleptiques, ces animaux ne sont toutefois pas véritablement hypersomnolents [9]. Les résultats obtenus chez l'animal doivent donc être interprétés avec prudence dans le contexte de la narcolepsie humaine.

Chez l'homme, une diminution sélective de la dopamine libre et de son principal métabolite, l'acide homovanillique, a été observée dans le liquide céphalorachidien de divers groupes de sujets hypersomniaques [10]. De plus, les psychostimulants utilisés pour traiter la SDE sont des agonistes de la transmission dopaminergique. Ces résultats suggèrent que l'hypersomnolence peut être associée à une diminution de la disponibilité biologique de la dopamine au niveau du système nerveux central. Quant à la cataplexie, on connaît l'effet thérapeutique de certains antidépresseurs tricycliques comme la clomipramine, dont les actions biologiques comprennent l'augmentation de la transmission sérotoninergique et le blocage de la transmission cholinergique au niveau du système nerveux central. Or, des bloquants spécifiques de la recapture de la sérotonine sans effet anticholinergique exerçent un effet antagoniste puissant sur la cataplexie sans affecter l'hypersomnolence [11]. Ceci suggère l'implication des circuits sérotoninergiques dans l'éta- 


\section{RÉFÉRENCES}

11. Montplaisir J, Godbout R. Serotoninergic reuptake mechanisms in the control of cataplexy. Sleep 1986 ; 9 : 275-9.

12. Billiard M, Cadilhac J. La narcolepsie. Rev Neurol (Paris); 17 : 220-6.

13. Kessler S. Genetic factors in narcolepsy. In : Guilleminault C, Dement WC, Passouant $\mathrm{P}$, eds. Narcolepsy. New York: Spectrum, 1976 : 285-302.

14. Kessler S, Guilleminault C, Dement WC A family study of 50 REM narcoleptics. Acta Neurol Scand 1974; 50 : 503-512.

15. BruhovaS, Roth B. Heredofamilial aspects of narcolepsy and hypersomnia. Schweiz Arch Neurol Neurochir Psychiatr $1972 ; 110$ : 45-54.

16. Honda Y, Asaka A, Tanimura M Furusho T. A genetic study of narcolepsy and excessive daytime sleepiness in 308 familie with a narcolepsy or hypersomnia proband In : Guilleminault C, Lugaresi E, eds. Sleep Wake Disorders: Natural History, Epidemio logy, and Long-Term Evolution. New York Raven Press, 1983 : 187-99.

17. Foutz AS, Mitler MM, Cavali-Sforza LL Dement WC. Genetic factors in canine narcolepsy. Sleep 1979; 1 : 413-22.

18. Imlah NW. Narcolepsy in identical twins J Neurol Neurosurg Psychiatry 1961 ; 24 : 158 60 .

19. Mamelak M, Caruso VJ, Stewart K. Nar colepsy: a family study. Biol Psychiatry 1979 ; $14: 821-34$.

20. Montplaisir J, Poirier G. Narcolepsy in monozygotic twins. Neurology 1987; 37 : 1089

21. Montplaisir J, Poirier G. Narcolepsy in monozygotic twins and non-genetic factors in narcolepsy. In: Chase MH, McGinty DJ, O'Connor C, eds. Sleep Research, vol, 16. Los Angeles: Brain Informations Service, 1987 396.

22. Juji T, Satake M, Honda Y, Doi Y. HLA antigens in Japanese patients with narcolepsy. All the patients were DR2 positive Tissue Antigens 1984; 24 : 316-19. blissement de la cataplexie. Par ailleurs, ce symptôme ne semble pas nécessairement secondaire à une modification neurochimique liée à la SDE, puisqu'environ $4 \%$ des cas rapportés dans la littérature ont développé la cataplexie avant la SDE [12].

Les études effectuées chez l'homme suggèrent également que les mécanismes impliqués dans l'hypersomnolence et la cataplexie sont différents. Du point de vue clinique, ils apparaissent à différents moments au cours de l'évolution de la maladie. De plus, les médicaments efficaces pour traiter l'hypersomnolence n'ont aucun effet sur la cataplexie et inversement les agents anti-cataplectiques n'ont pas d'effet sur l'hypersomnie de ces patients.

Un trouble du sommeil paradoxal. La narcolepsie peut être comprise comme un trouble de la régulation du sommeil paradoxal, dont les endormissements directs en sommeil paradoxal seraient l'épiphénomène. En effet tous les symptômes de la narcolepsie humaine représentent d'une certaine manière des manifestations dissociées d'éléments faisant partie de ce stade du sommeil. Par exemple, la cataplexie et les paralysies du sommeil pourraient traduire la mise en activité des mécanismes effecteurs responsables de l'inhibition motrice propre au sommeil paradoxal, alors que les hallucinations hypnagogiques correspondraient au matériel somato-sensoriel du rêve, lequel est étroitement associé à ce stade du sommeil.

Facteurs génétiques. L'étude des familles présentant plus d'un cas de narcolepsie suggère que cette maladie repose sur une base génétique. Près de $58 \%$ de nos patients rapportent la présence de SDE dans leur famille et $16 \%$ identifient clairement la narcolepsie chez un ou plusieurs membres de leur famille. Kessler [13, 14] ainsi que Bruhova et Roth [15] estimèrent que le risque de développer la SDE était respectivement 60 et 200 fois plus élevé dans la famille des narcoleptiques que dans la population générale. Les études familiales les plus récentes semblent indiquer un mode de transmission multifactoriel à deux seuils. Ce modèle propose que les sujets présentant une SDE partagent une même prédisposition génétique à l'hypersomnolence, l'expression phénotypique étant plus sévère dans la narcolepsie [16]. Ici encore, des différences importantes existent entre la narcolepsie animale et humaine, puisque chez les chiens «narcoleptiques » la maladie semble se transmettre selon un mode autosomique récessif [17].

L'étude de jumeaux monozygotes atteints de narcolepsie a parfois été utilisée dans la littérature scientifique comme argumentation en faveur d'une base génétique pour cette maladie. Toutefois, dans aucune étude antérieure d e jumeaux soi-dis a n t «concordants » pour la maladie, les deux jumeaux ne répondaient à la fois aux critères cliniques et électrographiques de la narcolepsie [18, 19]. Une investigation clinique, immunogénétique et électrophysiologique de deux couples de jumeaux monozygotes discordants pour la narcolepsie $[20,21]$ a permis de poser un diagnostic non équivoque de narcolepsie chez les propositi, mais leurs jumeaux n'avaient ni symptôme de narcolepsie, ni endormissement direct en sommeil paradoxal. Ces cas suggèrent fortement que les facteurs génétiques ne peuvent prédire à eux seuls l'apparition de la maladie, et que des facteurs extrinsèques sont nécessaires à son développement. Au cours des dernières années, les études immunogénétiques de la narcolepsie ont pris une ampleur considérable. Presque $100 \%$ des patients narcoleptiques sont porteurs de l'antigène HLA-DR2 alors que celui-ci ne se retrouve que chez $22 \%$ de la population caucasoïde. Ceci constitue l'association, entre un antigène HLA et une maladie, la plus forte jamais rapportée [22-24]. Cette association pourrait même être complète (100\%) si des critères diagnostiques plus stricts étaient utilisés 
pour sélectionner les sujets [25]. Les narcoleptiques négroïdes et mongoloïdes possèdent également cet antigène, ce qui suggère l'universalité de ce lien [26, 27]. Le gène contrôlant la fabrication de l'antigène HLA-DR2 est situé sur le petit bras du sixième chromosome. Bien qu'il semble exister sur ce chromosome un gène de susceptibilité à la narcolepsie, l'existence de quelques patients ne possédant pas l'antigène HLADR2 permet d'envisager que d'autres facteurs pourraient être impliqués. En effet, on sait que la narcolepsie peut être associée à une atteinte spécifique du système nerveux central à la suite de divers processus pathologiques, infectieux, traumatiques ou autres [23, 28].

L'antigène HLA-DR2 a également été associé à la sclérose en plaques et se retrouve chez environ 50 à $60 \%$ des patients atteints de cette maladie. Une étude immunogénétique et électrophysiologique de 70 cas de sclérose en plaques n'a toutefois pas permis de démontrer une association entre cette maladie et la narcolepsie [29].

Des études en cours ont pour but de comparer chez des sujets narcoleptiques et non narcoleptiques la structure moléculaire du gène HLA-DR2. Certains auteurs [27] ont observé des différences entre les deux groupes mais il est à notre avis trop tôt pour se prononcer sur cette question.

Facteurs environnementaux. L'apparition de somnolence diurne et de cataplexie est associée à des facteurs déclenchants particuliers chez environ $80 \%$ de nos malades. Dans de nombreux cas, la somnolence diurne est apparue plus de 20 ans avant le début de la cataplexie et en étroite relation temporelle avec des situations telles que la privation chronique de sommeil, le travail à horaires alternants, un deuil ou d'autres problèmes émotionnels, professionnels ou financiers. L'existence de facteurs associés au déclenchement a été rapportée dans la littérature [28]. Parkes et al. [30] ont noté que $15 \%$ de leurs $\mathrm{m} / \mathrm{s} n^{\circ} 4$ vol. 4 , avril 88 patients narcoleptiques avaient débuté la maladie de façon brutale, notamment à la suite d'une infection virale; ce phénomène $a$ été observé chez environ $6 \%$ de nos malades. Le cas le plus remarquable est celui d'un homme de 50 ans qui ne présentait aucune histoire antérieure de maladie neurologique et aucun symptôme de narcolepsie jusqu'au moment où il a éte piqué a la main par une mouche non infectieuse très commune au Québec (Tabanus $s p$.). Après avoir développé dans les jours suivants une réaction inflammatoire sévère, il éprouva une période de frissons intenses, sans qu'aucune hausse de sa température corporelle ne soit notée. Environ cinq jours plus tard, une somnolence diurne, des poussées incoercibles de sommeil et des accès de cataplexie apparurent simultanément. L'investigation électrophysiologique confirma le diagnostic de narcolepsie, suite à l'enregistrement de cinq endormissements directs en sommeil paradoxal. Ce patient était également porteur de l'antigène HLADR2 mais ne rapportait aucune histoire familiale de narcolepsie ou d'hypersomnie. Ce cas particulier soulève de nouveau la possibilité qu'un facteur extrinsèque puisse déclencher la maladie chez un individu génétiquement prédisposé.

\section{Vers un modèle} d'étiologie multifactorielle

Le regroupement des données présentées ci-dessus permet de présenter un modèle d'étiologie de la narcolepsie. La narcolepsie animale et humaine pourrait résulter d'une atteinte à des niveaux différents des structures nerveuses impliquées dans les manifestations du sommeil paradoxal. Dans la narcolepsie humaine, il y aurait une atteinte des structures diencéphaliques d'induction du sommeil, lesquelles sont également responsables de la coordination des différents éléments du sommeil paradoxal. Dans la narcolepsie animale, la maladie toucherait plutôt les sous-structures effectrices du sommeil paradoxal, situées au niveau du tronc cérébral. Cette distinction pourrait expliquer en partie l'absence d'hypersomnolence sévère chez les chiens narcoleptiques, alors que ce symptôme est prédominant dans la narcolepsie humaine. Cette hypothèse pourrait également rendre compte des différences observées au niveau de la cataplexie, laquelle est à la fois beaucoup plus fréquente (plusieurs heures chaque jour) et moins directement liée à la présence d'émotions spécifiques chez l'animal.

Quant au rôle du HLA-DR2 dans la maladie, une hypothèse pourrait être que cet antigène est différent ou modifié chez les narcoleptiques par rapport aux porteurs sains. L'étude de familles de narcoleptiques ne semble toutefois pas appuyer cette hypothèse. En effet, comme les antigènes HLA sont transmis en bloc des parents à leurs enfants, la molécule HLA-DR2 est vraisemblablement identique pour tous les membres d'une même famille. Or 30 membres de trois familles possédaient l'antigène HLA-DR2 et sept seulement $(20,3 \%)$ ont développé la narcolepsie [24]. Il est peu probable que seulement sept de ces 30 personnes apparentées aient pu posséder un antigène HLA-DR2 modifié. Cet argument est également valable pour les jumeaux monozygotes discordants.

La narcolepsie pourrait, d'autre part, ne pas être directement liée au HLA-DR2 mais plutôt à un gène encore inconnu qui lui serait adjacent sur le sixième chromosome et toujours transmis avec lui. Dans une telle hypothèse, une recombinaison génétique exceptionnelle faisant suite à un fractionnement entre leurs loci respectifs pourrait expliquer les quelques rares cas de narcolepsie typique ne possédant pas le HLADR2. Des études immunogénétiques détaillées de la famille de ces malades narcoleptiques non porteurs de l'antigène HLA-DR2 permettraient de préciser la pré- 


\section{RÉFÉRENCES}

23. Poirier G, Montplaisir J, Décary F, Momège D, Lebrun A. HI.A antigens in narcolepsy and idiopathic central nervous system hypersomnolence. Sleep $1986 ; 9$ : 153-8.

24. Montplaisir J, Poirier G. HLA in narcolepsy in Canada. In: Honda Y, et al. eds. $H L A$ in Narcolepsy. Berlin : Springer Verlag (sous presse).

25. Matsuki K, Honda Y, Juji T. Diagnostic criteria for narcolepsy and HLA-DR2 frequencies. Tissue Antigens 1987 ; 30 : 155-60.

26. Kramer RE, Dinner DS, Braun WE, Zachary AA, Teresi GA. HLA-DR2 and narcolepsy. Arch Neurol 1987 ; 44 : 853-5.

27. Honda Y, Juji T, Matsuki K, et al. HLADR2 and Dw2 in narcolepsy and in other disorders of excessive somnolence without cataplexy. Sleep 1986 ; 9 : 133-42.

28. Passouant $P$, Billiard $M$. The evolution of narcolepsy with age. In: Guilleminault $C$, Dement WC, Passouant P, eds. Narcolepsy. New York : Spectrum, 1976 : 179-96.

29. Poirier G, Montplaisir J, Dumont M, et al. Clinical and sleep laboratory study of narcoleptic symptoms in multiple sclerosis. Neurology 1987 ; 37 : 693-95.

30. Parkes JD, Langdon N, Lock C. Narcolepsy and immunity. Br Med J 1986; 292 : 359-60.

31. Palmbald J. Stress and immunologic competence: Studies in man. In : Ader R, ed. Psychoneuroimmunology. New York: Academic Press, 1981 : 229-57.

32. Gachelin G. Emotions et immunité. La Recherche 1986 ; 17 : 662-6.

33. Hors J. HLA et maladies. In : Dausset J, Pla M, eds. HLA, Complexe Majeur d'Histocompatibilité de l'Homme. Paris: Flamma244 sence ou non d'une recombinaison génétique et, secondairement, la localisation du gène prédisposant à la narcolepsie.

Les données présentées ci-dessus suggèrent que la narcolepsie est une maladie multifactorielle. On peut s'interroger sur l'interaction existant entre ces différents facteurs.

Il a été démontré par exemple qu'une période de privation de sommeil peut diminuer la réponse immunitaire chez l'homme et l'animal [31]. De plus, des études récentes [32] suggèrent un affaiblissement des réponses immunitaires chez l'homme lors d'un deuil, d'une souffrance psychique ou d'une dépression. Le lien entre les émotions et l'immunité pourrait être associé à la capacité qu'ont les cellules lymphoïdes thymiques d'intégrer des signaux émis par le système nerveux en réponse à des informations extrinsèques, modulant ainsi l'activité immunitaire.

Parallèlement, d'autres auteurs [33] ont attribué à l'antigène HLA-DR2 un rôle suppresseur de la réponse immunitaire. Par conséquent, des facteurs physiques ou psychologiques extrinsèques abaissant la réponse immunitaire, couplés à la présence d'un HLA-DR2 immunosuppresseur, pourraient entraîner le developpement de la narcolepsie. Le système immunitaire serait ainsi impliqué dans le développement de la narcolepsie, bien qu'il soit encore difficile d'en préciser le rôle. Des études exhaustives sur les anomalies immunitaires de patients narcoleptiques sont donc nécessaires. Il est également important de poursuivre les études sur les mécanismes neurochimiques responsables des différents symptômes de la narcolepsie et de mieux comprendre les relations entre la conformation génétique particulière à la narcolepsie et le fonctionnement des différents systèmes neurochimiques qui y sont impliqués. Le modèle étiologique de la narcolepsie pourrait ainsi permettre d'élucider la complexité des mécanismes qui peuvent interagir pour produire d'autres maladies

\section{Summary}

Narcolepsy is a neurological disorder characterized by two major symptoms, namely excessive daytime sleepiness and cataplexy. Neurochemical and pharmacological studies suggest that different mechanisms are involved in the physiopathology of these symptoms. In human narcolepsy, excessive daytime sleepiness seems to result from a decreased dopaminergic activity in the central nervous system while decreased serotoninergic activity may lead to the development of cataplexy. The specificity of the clinical features and the presence of an objective diagnostic criteria made possible the discovery of a specific genetic marker. While, nearly all narcoleptic patients carry the HLA-DR2 antigen, it is not found to be elevated in other groups of hypersomniac patients. On the other hand, several environmental factors are found to be closely associated in time with the development of the illness. Thus, narcolepsy appears more and more as a multifactorial disease probably involving the immune system. Further research will be necessary to specify the genetic defect, the neurotransmitters involved, the nature of environmental factors, and the mode of interaction of these elements.

\section{Remerciements}

Ces recherches sont subventionnées par le conseil de recherches médicales du Canada, le fonds de la recherche en santé du Québec (FRSQ), et la Fondation Sacré-Cour. Nous tenons à souligner les contributions scientifiques de Francine Décary, André Lebrun et Daniele Momège.

\section{TIRÉS A PART}

J. Montplaisir : centre d'étude du sommeil, hôpital du Sacré-Cœur, 5400 bd Gouin Ouest, Montréal (Québec), H4J 1C5, Canada. 